OPEN ACCESS

Edited by:

Maria S. Guillem

Universitat Politècnica de València,

Spain

Reviewed by:

Matthijs Cluitmans,

Maastricht University, Netherlands

Crystal M. Ripplinger,

University of California, Davis,

United States

Jess D. Tate,

University of Utah, United States

*Correspondence:

Erick Andres Perez Alday

perezald@ohsu.edu

Michael A. Colman

m.a.colman@/eeds.ac.uk

Specialty section:

This article was submitted to

Cardiac Electrophysiology,

a section of the journal

Frontiers in Physiology

Received: 13 September 2018

Accepted: 07 March 2019

Published: 05 April 2019

Citation:

Perez Alday EA, Whittaker DG Benson AP and Colman MA (2019) Effects of Heart Rate and Ventricular

Wall Thickness on Non-invasive

Mapping: An in silico Study.

Front. Physiol. 10:308.

doi: 10.3389/fphys.2019.00308

\section{Effects of Heart Rate and Ventricular Wall Thickness on Non-invasive Mapping: An in silico Study}

\author{
Erick Andres Perez Alday ${ }^{1 *}$, Dominic G. Whittaker ${ }^{2}$, Alan P. Benson ${ }^{2}$ and \\ Michael A. Colman ${ }^{2 *}$ \\ ${ }^{1}$ Knight Cardiovascular Institute, Oregon Health \& Science University, Portland, OR, United States, ${ }^{2}$ School of Biomedical \\ Science and Multidisciplinary Cardiovascular Research Centre, University of Leeds, Leeds, United Kingdom
}

Background: Non-invasive cardiac mapping-also known as Electrocardiographic imaging (ECGi) - is a novel, painless and relatively economic method to map the electrical activation and repolarization patterns of the heart, providing a valuable tool for early identification and diagnosis of conduction abnormalities and arrhythmias. Moreover, the ability to obtain information on cardiac electrical activity non-invasively using ECGi provides the potential for a priori information to guide invasive surgical procedures, improving success rates, and reducing procedure time.

Previous studies have shown the influence of clinical variables, such as heart rate, heart size, endocardial wall, and body composition on surface electrocardiogram (ECG) measurements. The influence of clinical variables on the ECG variability has provided information on cardiovascular control and its abnormalities in various pathologies. However, the effects of such clinical variables on the Body Surface Potential (BSP) and ECGi maps have yet to be systematically investigated.

Methods: In this study we investigated the effects of heart size, intracardiac thickness, and heart rate on BSP and ECGi maps using a previously-developed 3D electrophysiologically-detailed ventricles-torso model. The inverse solution was solved using the three different Tikhonov regularization methods.

Results: Through comparison of multiple measures of error/accuracy on the ECGi reconstructions, our results showed that using different heart geometries to solve the forward and inverse problems produced a larger estimated focal excitation location. An increase of $\sim 2 \mathrm{~mm}$ in the Euclidean distance error was observed for an increase in the heart size. However, the estimation of the location of focal activity was still able to be obtained. Similarly, a Euclidean distance increase was observed when the order of regularization was reduced.

For the case of activation maps reconstructed at the same ectopic focus location but different heart rates, an increase in the errors and Euclidean distance was observed when the heart rate was increased. 


\begin{abstract}
Conclusions: Non-invasive cardiac mapping can still provide useful information about cardiac activation patterns for the cases when a different geometry is used for the inverse problem compared to the one used for the forward solution; rapid pacing rates can induce order-dependent errors in the accuracy of reconstruction.
\end{abstract}

Keywords: ECGi, non-invasive mapping, body surface potential, heart rate, cardiac hyperthrophy

\section{INTRODUCTION}

Cardiovascular disease is a major contributor to reduced quality of life and mortality worldwide (Benjamin et al., 2017). Cardiac conditions such as heart failure, myocardial infarction, and hypertrophic/dilated cardiac myopathy are related to electrical dysfunction (i.e., arrhythmia) and typically result in reduced cardiac output. Diagnosis and treatment of these conditions presents a significant healthcare challenge, in part due to their dual electrophysiological-structural components. Shortand long-term adaptation of cardiac structure and ion channel expression, which includes reversible and irreversible remodeling associated with disease, further compounds the challenge. For example, cardiac hypertrophy, which is an important risk factor of heart failure and sudden cardiac death (Vriesendorp et al., 2015), is characterized by abnormal thickening of the heart muscle, usually resulting from increases in cardiac cell size, in order to compensate for inhibited contractile performance (Shimizu and Minamino, 2016). The particular manifestation of electrical dysfunction may therefore vary over the time-course of the condition; the ability to accurately map the electrical activity of the heart non-invasively over this whole period can offer significant advantages for the long-term management of such conditions.

Electrocardiographic imaging (ECGi) is a novel, painless and (relatively) economic method to map the electrical activation and repolarization patterns of the heart (Ghosh et al., 2011; Alday et al., 2016; Bear et al., 2016; Perez Alday et al., 2016; Zhang et al., 2016), and presents the possibility to better understand cardiac excitation patterns and provide a priori information to guide invasive surgical procedures, improving success rates and reducing procedure time (Silva et al., 2009; Dubois et al., 2015; Zhang et al., 2016). Based on solving the inverse problem of electrocardiography, with the heart acting as an electrical source inside the volume conductor of the body, ECGi aims to reconstruct the electrical activity on the surface of the heart using body surface potential (BSP) maps obtained from torso surface multi-array electrocardiogram (ECG) systems (Macfarlane et al., 2010; Rudy, 2013; Perez-Alday et al., 2017b). It depends on 3D heart and torso structures and therefore requires reconstructions of patients' cardiac and torso anatomy, which are typically acquired using the clinical imaging technologies of Magnetic Resonance Imaging (MRI) or Computed Tomography (CT). Due to the expense of these modalities, it may not be desirable to attain structural information from a patient repeatedly over the course of structural adaptions. However, the potential impact of using out-of-date structural information when performing ECGi is unclear.
In addition, previous studies have shown the influence of clinical variables, such as respiration (Langley et al., 2010; Baumert et al., 2013), body composition, (Zemzemi et al., 2015), and heart rate and body position (Appel et al., 1989; Goldenberg et al., 2006) on the ECG measurement. Based on these insights, adjusted ECG parameters (e.g., corrected QT interval) have improved the detection of patients at increased risk of cardiac arrhythmias (Kabir et al., 2016). It follows that such variables may also influence interpretation of BSP and ECGi data, but the nature of these relationships have yet to be systematically investigated.

The aim of this study was therefore to assess the effect of varying cardiac structure and electrical pacing rate on the accuracy of ECGi reconstructions. An in silico approach was used to provide clean and controllable data to compare reconstructions attained at multiple pacing rates and with underlying hypertrophic and dilated cardiac anatomy under sinus rhythm and ectopic focal excitation.

\section{METHODS}

The in silico approach utilized idealized, electrophysiologically heterogeneous human bi-ventricle models to simulate electrical excitation in control, dilated and hypertrophied conditions (sections "Virtual Bi-ventricle Models" to "Ventricular Simulation Protocols"). Ventricular activation was then combined with a heterogeneous torso model and the forward problem was solved to produce simulated BSP maps (section "Simulated Body Surface Potential"). The inverse solution, using multiple regularization approaches, was applied to the simulated BSP maps in order to produce ECGi epicardial potential reconstructions and compute activation patterns (section "Inverse Solution"). Multiple measures were used to quantify and compare results obtained under the different conditions (section "Analysis Methods").

\section{Virtual Bi-Ventricle Models}

Idealized human bi-ventricle geometries were constructed as structured finite difference grids, wherein the left and right ventricles (LV and RV, respectively) were modeled as thick- and thin-walled truncated ellipsoids, respectively. A control (normal) geometry was constructed in order to have physiologicallyaccurate ventricular wall thicknesses (12-15 and 3-5 $\mathrm{mm}$ for LV and RV, respectively; Ho and Nihoyannopoulos, 2006; Ho, 2009) and volumes ( 150-210 mL for the LV in human males; Alfakih et al., 2003; Clay et al., 2006), and the overall size and ventricular curvature were qualitatively matched against multiple 
existing human ventricle datasets (Seemann et al., 2006; Benson et al., 2011; Keller et al., 2011). From this, two more geometries were created by increasing either the wall thickness or the short axis diameter by $50 \%$. In total three cases were considered: (i) normal, (ii) thick-walled (hypertrophied), and (iii) dilated ventricles (Figure 1A). A spatial resolution of $\Delta x=\Delta y=\Delta z$ $=0.5 \mathrm{~mm}$ was used, which gave $\sim 2 \times 10^{6}$ nodes in tissue, to facilitate high-throughput generation of multiple datasets for BSP and ECGi analysis. Measurements of the LV volume, LV wall thickness, and RV wall thickness from the developed geometries are given in Table $\mathbf{1}$.

In each case, a simple ruled-based model was implemented to assign myocardial fiber orientations (Figure 1Ci) using a standard approach based on rules proposed by Streeter et al. (1969). A value of the helix angle, $\alpha$, was assigned to each node of the grid, given by

$$
\alpha=R(1-2 d)^{n}
$$

where $R$ is the transmural rotation (varying from $+R$ at the endocardium to $-R$ at the epicardium), $d$ is the normalized transmural depth (varying from 0 at the endocardium to 1 at the epicardium), and $n$ determines the transmural variation in helix angle (e.g., $n=1$ is linear, $n=3$ is cubic). For all simulations in this study, $R$ was set to $60^{\circ}$, giving a transmural rotation in helix angle of $120^{\circ}$, similar to that observed in existing human ventricular datasets (Seemann et al., 2006; Benson et al., 2010), and $n$ was set to 1 (Benson et al., 2008). The transverse angle was assumed to be $0^{\circ}$ as it has been shown to be constantly around $0^{\circ}$ throughout the ventricles (Seemann et al., 2006), and no sheetlet structure was incorporated, as this has been suggested to show great variability between hearts (Benson et al., 2008). A small degree of smoothing was applied where the right ventricle joins the ventricular septum, to ensure a smooth transition in helix angles.

\section{Single Cell Model of Human Ventricles}

To simulate the action potential (AP) of human ventricular myocytes, the 2006 version of the Ten Tusscher et al. model was used (Ten Tusscher and Panfilov, 2006), which accounts for distinct electrophysiological differences in cells from the

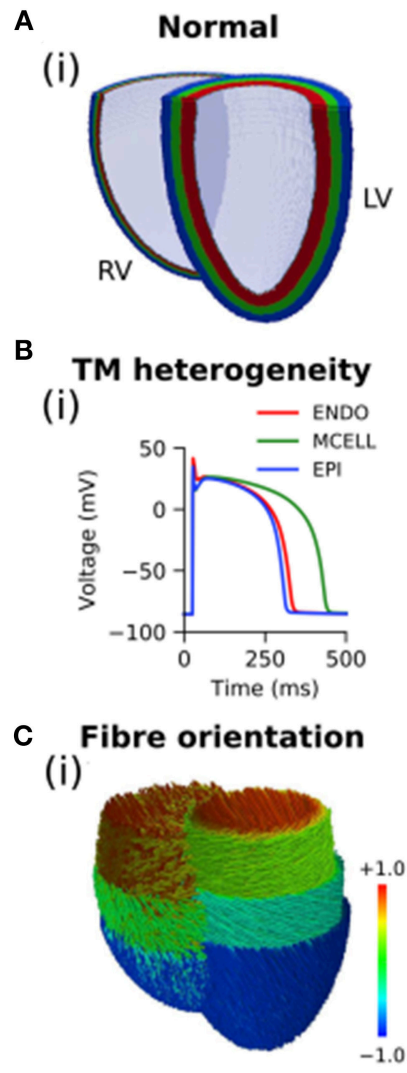

(ii)

Thick-walled

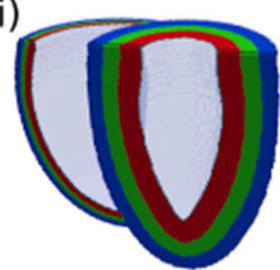

(ii)
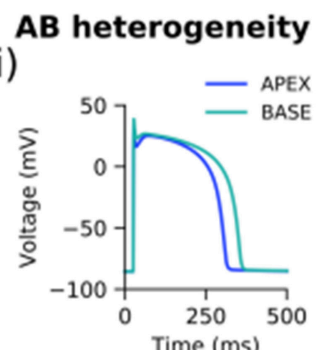

Heart-torso model

(ii)

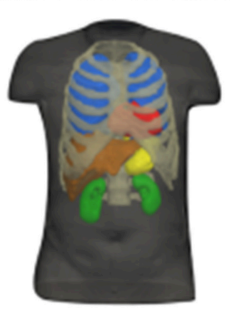

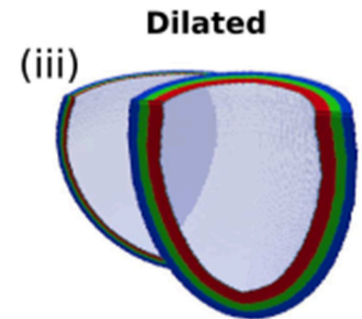

Short AP

(iii)

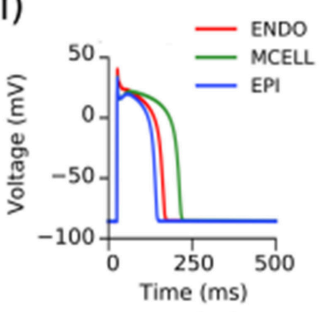

(iii)

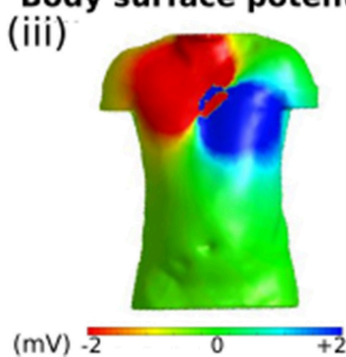

FIGURE 1 | Computational models of the human ventricles and torso. (A) An open view of geometries representing (i) normal/control, (ii) thick-walled, and (iii) dilated human ventricles with the epicardial (blue), mid-myocardial (green), and endocardial (red) segmented regions shown. (B) Single cell ventricular action potentials representing (i) transmural (TM) heterogeneity in cells from the endocardium (ENDO), mid-myocardium (MCELL), and epicardium (EPI), (ii) apico-basal (AB) heterogeneity (shown for EPI cells), and (iii) short single cell action potentials used in this study. All models are uncoupled single cells paced at a cycle length of $1,000 \mathrm{~ms}$. (C) (i) Fiber orientation (normalized z component of primary fiber; red and blue indicate parallel to the long axis of the heart from apex to base, green indicates perpendicular to the long axis of the heart); (ii) Heart-torso model used to compute the (iii) body surface potential. 
TABLE 1 | A summary of dimensions in developed idealized ventricular geometries.

\begin{tabular}{lrcr}
\hline & Control & Thick-walled & Dilated \\
\hline LV wall volume $(\mathrm{mL})$ & 196.56 & 249.77 & 255.29 \\
LV wall thickness $(\mathrm{mm})$ & 12.00 & 18.00 & 12.00 \\
RV wall thickness $(\mathrm{mm})$ & 4.00 & 6.00 & 4.00 \\
\hline
\end{tabular}

ventricular endocardium (ENDO), mid-myocardium (MCELL), and epicardium (EPI; Figure 1Bi). The bi-ventricle models were segmented into 40\% ENDO, 30\% MCELL, and 30\% EPI cells (Figure 1A), similar to previously used ratios (Adeniran et al., 2011, 2017). The existing transmural heterogeneity was increased by adjusting the ENDO:EPI:MCELL ratio of rapid delayed rectifier potassium current, $I_{\mathrm{Kr}}$, maximal conductance to 1.0:1.6:1.0 (Adeniran et al., 2011; Whittaker et al., 2017). This was based on transmural measurements of hERG mRNA expression (Szabó et al., 2005), and was necessary to reproduce the longer AP of ENDO compared to EPI cells (Glukhov et al., 2010; Boukens et al., 2015). Furthermore, a linear gradient in the conductance of transient outward potassium current, $I_{\text {to }}$, and slow delayed rectifier potassium current, $I_{\mathrm{Ks}}$, was introduced along the apexbase (AB) axis (Keller et al., 2011; Alday et al., 2016). Briefly, maximal conductance of $I_{\mathrm{to}}$ and $I_{\mathrm{Ks}}$ were reduced by a maximum of $50 \%$ in basal cells relative to apical cells in order to reproduce apico-basal heterogeneity (Figure 1Bii), giving a roughly $50 \mathrm{~ms}$ longer AP duration in basal cells than in apical cells (Szentadrassy et al., 2005). The maximal conductance of current $x$ from cell type $y, g_{x, y}$, was given by

$$
\begin{aligned}
g_{x, y} & =g_{\text {Base }, y}+\left(g_{\text {Apex } y}-g_{\text {Base }, y}\right) \cdot f_{A B}, \\
f_{A B} & =\frac{z-z_{\text {Base }}}{z_{\text {Apex }}-z_{\text {Base }}},
\end{aligned}
$$

where $g_{\text {Apex }, y}$ and $g_{\text {Base }, y}$ are maximal values of the conductance of cell type $y$ at the apex and base, respectively, $f_{\mathrm{AB}}$ is a gradient factor which depends linearly on the value of the $z$ co-ordinate which lies along the $\mathrm{AB}$ axis (varying from 1 at the apex to 0 at the base), and $z_{\text {Base }}$ and $z_{\text {Apex }}$ are the values of the $z$ coordinate at the apex and base, respectively. No electrophysiological differences were incorporated between the LV and RV (Keller et al., 2011).

\section{Modeling Action Potential Propagation}

The monodomain equation was used to describe the propagation of APs in the bi-ventricle geometries:

$$
\frac{\partial V}{\partial t}=\nabla(\boldsymbol{D} \nabla V)-\frac{I_{\text {ion }}}{C_{\mathrm{m}}},
$$

where $V$ is the transmembrane voltage, $\mathbf{D}$ is the global conductivity tensor, $I_{\text {ion }}$ is the total ionic current, and $C_{\mathrm{m}}$ is the membrane capacitance. Equation (4) was solved numerically using a finite-difference PDE solver based on the explicit forward Euler method, using an operator splitting technique and an adaptive time step with minimum and maximum time steps of $\Delta t_{\min }=0.02 \mathrm{~ms}$ and $\Delta t_{\text {max }}=0.2 \mathrm{~ms}$, respectively (Benson et al., 2010). As axially-symmetric anisotropy was assumed, two principal values of the diffusion coefficient were required: $D_{\|}$, the longitudinal value of the conductivity which describes propagation in the fiber direction, and $D_{\perp}$, the transverse value, which describes propagation orthogonal to fibers. The diffusion tensor can thus be written as

$$
\mathbf{D}=D_{\perp} \mathbf{I}+\left(D_{\|}-D_{\perp}\right) \mathbf{A} \boldsymbol{A}^{\mathrm{T}}
$$

where $\mathbf{I}$ is the identity matrix, $\mathbf{A}$ is a unit vector giving the fiber direction, and $\mathbf{A}^{\mathrm{T}}$ is the transpose of $\mathbf{A}$.

The longitudinal value of the conductivity, $D_{\|}$, was set to 0.18 $\mathrm{mm}^{2} \mathrm{~ms}^{-1}$ in this study, which gave a conduction velocity of 70 $\mathrm{cms}^{-1}$ in the fiber direction (Benson et al., 2007), in agreement with experimental measurements of conduction velocity along fibers in human ventricular tissue (Taggart et al., 2000). An anisotropic conductivity ratio of $D_{\|}: D_{\perp}=4: 1$ was used (Benson et al., 2007; Whittaker et al., 2017).

\section{Ventricular Simulation Protocols}

Sinus rhythm activation of the ventricles was elicited by stimulating a series of 28 localized patches (with diameters of $\sim 9$ $12 \mathrm{~mm}$ ) in quick succession along the endocardial wall (stimulus amplitude and duration $-52 \mathrm{pA} / \mathrm{pF}$ and $1 \mathrm{~ms}$, respectively, where the wavefront was initiated in the intra-ventricular septum before spreading from apex to base throughout the left and right ventricles. This gave a total activation time of $\sim 65 \mathrm{~ms}$ in the control geometry, in good agreement with the classic results of Durrer et al. (1970). For studying the effects of ectopic activity in the ventricles, four prescribed locations which could be easily identified in each of the geometries were chosen as "ectopic stimulus" sites: (i) the right ventricular lateral wall (RV-LAT), (ii) the intra-ventricular septum (SEP), (iii) the left ventricular lateral wall (LV-LAT), and (iv) the left ventricular apex (LV-Apex). In each case, localized $-52 \mathrm{pA} / \mathrm{pF}$ stimuli of $1 \mathrm{~ms}$ duration were applied over $5 \mathrm{~mm}$.

\section{Simulated Body Surface Potential}

The ventricular model was placed into a previously developed biophysically-detailed computational three-dimensional hearttorso model which accounts for the distinct structures of the lungs, liver, blood masses, stomach, spleen, kidneys, ribs and spinal cord, and the respective electrical conductivities (PerezAlday et al., 2015) (Figure 1Cii). This model has been previously used to develop an algorithm to diagnose atrial ectopic origin from multi lead ECG systems and ventricular ischemia (Alday et al., 2016; Perez-Alday et al., 2017a). Details of the torso model development, validation and simulation protocols can be found in Perez-Alday et al. (2015). Briefly, the heart-torso algorithm previously developed was used to solve the forward problem and obtain BSP maps (Figure 1Ciii) in each of the cases. The potential on the surface of the body was obtained from the 3D ventricular model using Salu's approach (Salu, 1980), utilizing the Boundary Element Method and Green's identities to solve the Poisson equation (Macfarlane et al., 2010). 


\section{Inverse Solution}

An inverse solution was developed, extending previously published preliminary work (Alday et al., 2016). Briefly, and based on prior work from Ramanathan and Rudy (2001), surface to surface torso-heart matrix was calculated using Barr's approach, where an equivalent potential distribution on a closed surface is used to build the homogenous heart-torso matrix (Barr et al., 1977); note therefore that, whereas the forward problem is solved on a heterogeneous torso, the inverse solution is provided on a homogeneous torso model. From the BSP maps, a previously developed inverse problem algorithm using Tikhonov regularization using Generalized Single Value Decomposition (GSVD) numerical approach was used to obtain the activation on the surface of the heart (Hansen, 1998). The potentials on the surface, $x$, were obtained by solving Equation (6):

$$
x=\min _{x}\left\{\|Z x-y\|_{2}+\lambda^{2}\|R x\|\right\},
$$

where $Z$ is the transfer matrix, $y$ represents the BSP vector, $\lambda$ is the regularization parameter obtained using the L-curve (Hansen, 1992), and $R$ is the regularization operator. Zero (Identity matrix, $R=\mathrm{I}$ ), First (Gradient operator, $R=\nabla$ ), and Second (Laplace operator, $R=\Delta$ ) order Tikhonov were used to regularize the solution. The GSVD technique was used to solve Equation (6) in each case.

As an ill-posed problem, noisy signals can have an important effect on the reconstructed maps. Whereas it is common in modeling studies of ECGi to include additional white noise, this was not performed in this study for the bulk of our analysis. Please see "Discussion: Limitations" for further details on the inclusion of noise and its impact.

\section{Analysis Methods}

Epicardial potentials were reconstructed from the BSP obtained at each instant of time for each geometry and activation case. Activation maps were calculated by computing maximal negative slope at each node at each time step (Gage et al., 2017). An example of original and reconstructed epicardial potential snapshots and the corresponding activation maps is shown in the Section S1 in Supplementary Material. To quantify the differences between the BSP and reconstructed activation maps for each of the geometry cases, three difference methods were used (Bear et al., 2015, 2018b):

1) Voltage root mean squared (RMS):

$$
R M S=\sqrt{\frac{\sum_{i=1}^{N} \varnothing_{i}^{2}}{N}} ;
$$

2) Relative RMS error (rRMSe):

$$
r R M S e=\sqrt{\frac{\sum_{i=1}^{N}\left(\varnothing_{i}^{\prime}-\varnothing_{i}\right)^{2}}{\sum_{i=1}^{N}\left(\varnothing_{i}^{\prime}\right)^{2}}}
$$

3) Pearson correlation coefficient (PCC):

$$
P C C=\frac{\sum_{i=1}^{N}\left(\varnothing_{i}{ }^{\prime}-\varnothing_{i}\right)\left(\varnothing_{i}{ }^{\prime}-\varnothing_{i}\right)}{\sqrt{\sum_{i=1}^{N}\left(\varnothing_{i}{ }^{\prime}-\varnothing_{i}\right)^{2}\left(\varnothing_{i}{ }^{\prime}-\varnothing_{i}\right)^{2}}},
$$

where $N$ is the number of elements in the mesh (torso or epicardial elements), $\varnothing$ is the potential reconstructed or measured and $\varnothing^{\prime}$ is the original simulated potential, while $\bar{\varnothing}$ and $\bar{\varnothing}^{\prime}$ are the mean potential values across all elements of the mesh. RMS gives an estimation of the variability of the signal. rRMSe gives an estimation of the variability between two methods. PCC is the measure of the correlation between two variables. The analysis was performed at each temporal snapshot of the ventricular activation.

To investigate the focus location accuracy of the inverse solutions, the Euclidean distance (ED) was calculated at the center of the earliest activation: ||$E D||=\sqrt{\left(r^{\prime}-r\right)^{2}}$, where $r$ is the center of activation of the reconstructed potential in the $3 \mathrm{D}$ Euclidean space and $r^{\prime}$ is the center of activation of the original simulated data. The Euclidean distance was calculated for all the ectopic cases and a median value is reported in this study.

\section{Investigating the Effect of Using the Incorrect Geometry for ECGi}

The impact of using only an initial patient anatomical reconstruction when performing ECGi, which doesn't capture any structural remodeling which may have occurred between the time of the scan and any present measurements, was investigated: Ectopic ventricular activation was simulated on all three geometries (control, thick-walled, and dilated; section "Virtual Bi-ventricle Models") and used to solve the forward problem and produce BSP maps; the ECGi reconstruction was performed using only the control geometry, representing the initial patient scan. Quantification of errors and correlations were performed by comparing the reconstruction with the control geometry activation for each matched ectopic location, such that geometrical differences don't have to be accounted for.

\section{Investigating the Effect of Heart Rate on ECGi}

The effect of heart rate on the epicardial reconstructions obtained using the three Tikhonov regularization methods was assessed for focal excitations using the control geometry paced at basic cycle lengths (BCL) of 1,000, 750, 500, 300, and $150 \mathrm{~ms}$ [corresponding to pacing rates of $60,80,120,200$, and 400 Beats per Minute (BPM), respectively]. Shortening of the AP (Figure 1Biii) induced by a five-fold increase in the conductance of $\mathrm{I}_{\mathrm{Kr}}$ and $\mathrm{I}_{\mathrm{Ks}}$ was employed to sustain the most rapid excitation rate $(\mathrm{BCL}=150 \mathrm{~ms})$. All other data were produced using the control AP models.

\section{RESULTS}

First, the impact of the different geometries (control, thickwalled, and dilated) on simulated BSP under control pacing conditions were compared to illustrate recapitulation of activation pattern and ECG differences observed under these conditions in the in silico framework (section "Simulated Body Surface Potential Under Different Conditions"). Then, the potential errors induced by using out-of-date and inaccurate cardiac anatomical reconstructions when performing ECGi was 
assessed (section "Effects of Wall Thickness and Heart Size on Non-invasive Cardiac Maps"). Finally, we investigated the effect of heart rate on the accuracy of reconstructed activation patterns using the different regularization approaches (section "Effects of Heart Rate on Non-invasive Cardiac Maps").

\section{Simulated Body Surface Potential Under Different Conditions}

The effects of the different geometries on the BSP were quantified by comparing the thick-walled and dilated geometries vs. the control during ventricular activation (Figure 2). Small differences were observed in the BSP maps at different instants of time (Figure 2A), quantitative measurements are plotted for comparison. A similar RMS was obtained for the three cases which produced relatively small rRMSe values (Figure 2B). However, the largest values were observed early during the activation sequence (first $75 \mathrm{~ms}$ ). A good agreement between the signal was observed for both cases (average PCC $>0.8$ ), however, at mid activation time (between 125 and $175 \mathrm{~ms}$ ) the values dropped significantly, with the dilated condition resulting in the smallest correlation.

\section{Effects of Wall Thickness and Heart Size on Non-invasive Cardiac Maps}

Data are illustrated for a single ectopic site only (RV-LATFigure 3) and summarized for all sites (Table 2). During the initial excitation phase $(75 \mathrm{~ms})$, similar small RMS and rRMSe values were observed for the three cases (Figure 3). During the mid and later activation times, RMS and rRMSe values were more dependent on the order of the regularization than the geometry, with First and Second order giving the smallest errors. Similarly, PCC values were considerably larger using First and Second order compared to the Zero order, and the Zero order displayed the most unique and geometry-dependent temporal evolution. The general increase in correlation over the time of the activation sequence is attributed to the increase in area of active tissue. RMS, rRMSe, and PCC were similar for all three heart geometries, although in general the control geometry exhibited the smallest errors and largest correlation and the dilated geometry exhibited the largest errors and smallest correlation (Figure 3; Table 2).

The calculated ED, measuring the error in correlation between real and identified focus location, varied for each geometry using the three Tikhonov methods (Figure 4A). Smaller values were observed for the Second order method (compared to Zero and First) and the control geometry (compared with thick and dilated, with dilated giving the largest values). However, the differences observed between geometries was less significant than that between methods.

\section{Effects of Heart Rate on Non-invasive Cardiac Maps}

The ED was calculated and compared for each different heart rate and Tikhonov method (Figure 4B). The Second order method in all the cases produced the smaller ED values. A marked increase in the ED was observed when the heart rate was increased for all methods, which also resulted in convergence of the solutions obtained using the different methods at the most rapid rate.

The reconstructed activation patterns were compared across the different pacing rates; illustrative data for the LV-LAT site are shown in Figure 5 and data from all ectopic sites are summarized in Table 3 . At the slowest pacing rates $(\mathrm{BCL}=1,000$ and $750 \mathrm{~ms}$ ), corresponding to normal heart rates in healthy patients (60 and $80 \mathrm{BPM}$, respectively), the Zero order method resulted in the larger rRMSe values and lower PCC values and contained the most noise. Both the First and Second order methods resulted in lower rRMSe values and larger PCC values over the temporal range of excitation (Figure 5-BCL $=1,000$ and $750 \mathrm{~ms}$ ), with the Second order in general performing the best, in congruence with the ED values (Figure 4B). The PCC for all methods in general increased over the time of the activation. At the most rapid rates (Figure $5-\mathrm{BCL}=300$ and $150 \mathrm{~ms}$ ), the temporal evolution of the PCC for First and Second order reversed, decreasing over the activation time, whereas the Zero order remained largely flat. The initial larger correlation for First and Second order compared to the slow pacing rates did not correspond to small ED and therefore was not a result of accurate reconstruction of the initial phase of excitation. The differences between the methods decreased at these rapid rates, largely due to an increase in the errors associated with First and Second order with no corresponding change to the Zero order solution (Figure 5, BCL $=150$ and $300 \mathrm{~ms}$ ). In all conditions, the Zero order approximation presented the most noise, but the reconstruction at the rapid excitation rates was more stable and comparable with the Second and First order (Table 3 ).

\section{DISCUSSION}

\section{Summary}

In this study, we used an in silico approach to evaluate the impact of different ventricular anatomical morphologies and heart rate on the accuracy of epicardial reconstructions attained through the application of the inverse solution to the BSP. We have demonstrated that the different cardiac anatomical states resulted in small but measurable differences in the BSP (Figure 2). Furthermore, we demonstrated that differences between actual underlying cardiac anatomy (i.e., the heart model on which electrical activation was simulated) and the reconstructed anatomy (i.e., the heart model on which the inverse solution was applied) led to errors in the reconstruction of both epicardial potential maps and activation patterns (Figure 3; Table 2). However, the location of the ectopic focal excitation was still largely correctly estimated, even with the incorrect geometry used for reconstruction (Figure 4). Moreover, we have demonstrated an important heart rate dependency of the correlation coefficients and reconstruction errors (Figure 5; Table 3). In general, the Second order regularization approach produced the smallest errors and largest correlation.

\section{Clinical Importance}

ECGi is a powerful and rapidly developing approach to noninvasively map patients' cardiac electrical activity in the clinic. The method aims to overcome some of the numerous challenges 
A
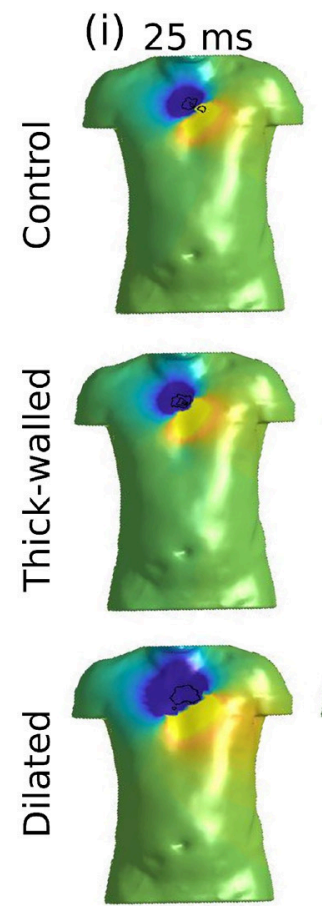

B

(i) (ii) $75 \mathrm{~ms}$
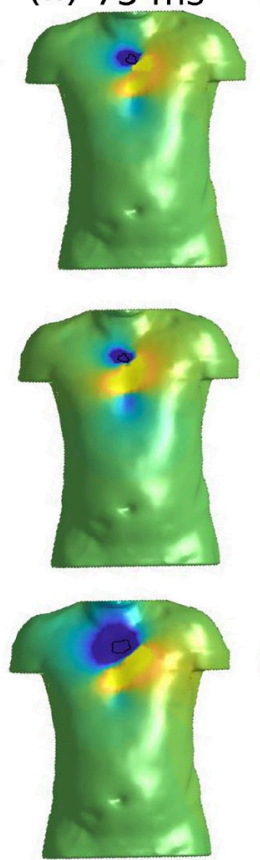

(ii)
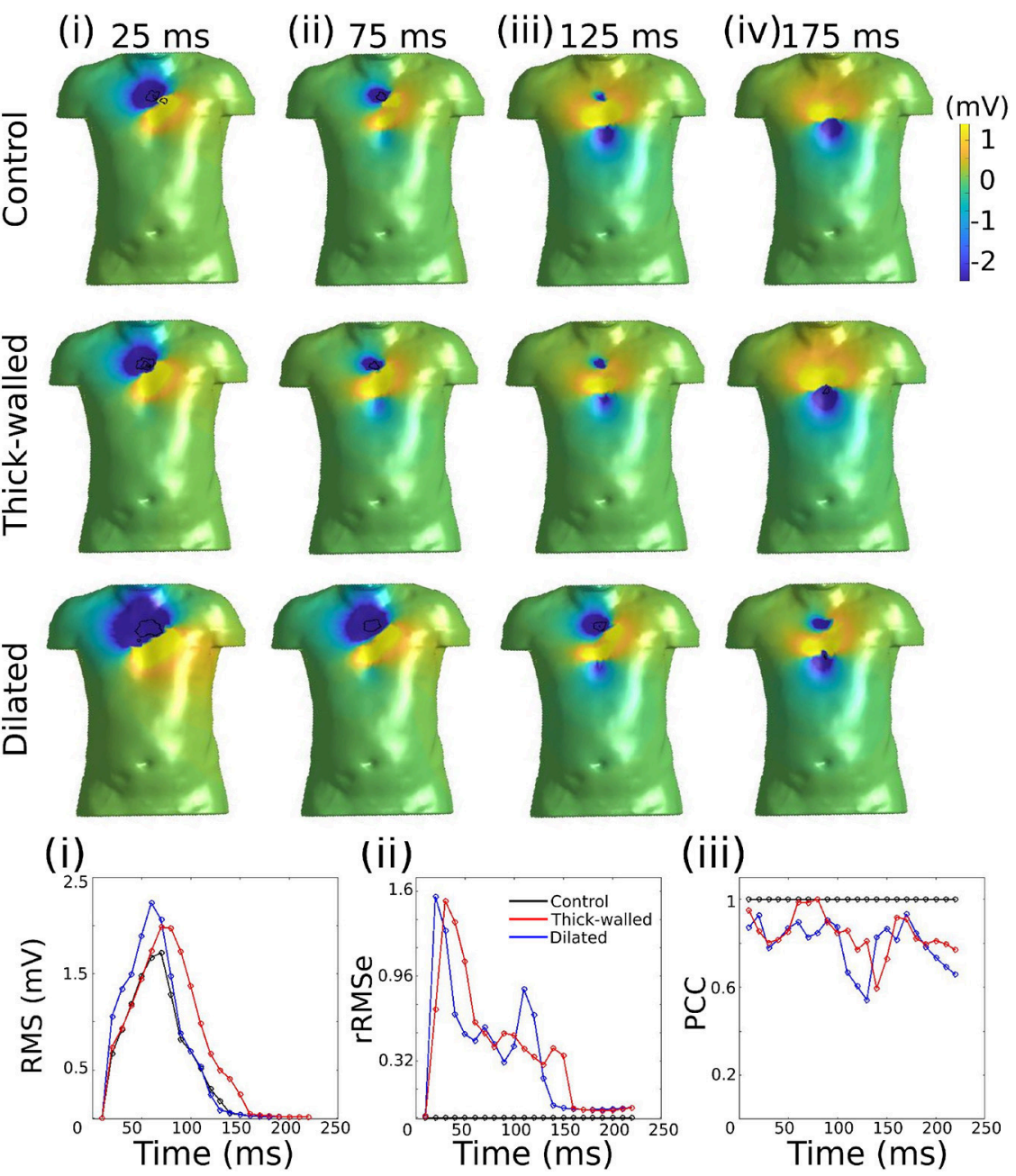

(iii)

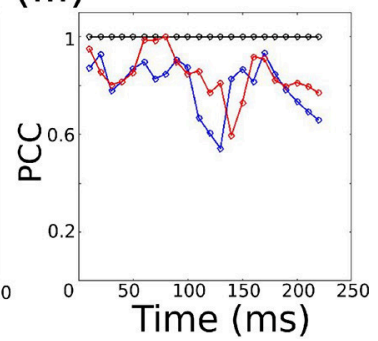

FIGURE 2 | Comparison of simulated BSP obtained from the three different geometries during ventricular activation. (A) BSP obtained for control case, the thick-walled and dilated geometry during simulated ectopic activation initiated in the right ventricular lateral wall (RV-LAT) at different instants of time: (i) 25 ms, (ii) $75 \mathrm{~ms}$, (iii) $125 \mathrm{~ms}$, and (iv) $175 \mathrm{~ms}$. (B) (i) RMS for each case and (ii) rRMSe and (iii) PCC calculated vs. the control case.

related to effective non-invasive characterization of human anatomy and electrophysiology. Previous studies have shown the usefulness of this non-invasive method to provide information to guide ablation procedures (Dubois et al., 2015; Rodrigo et al., 2017) and identify potential patients for whom cardiac resynchronization therapy would be successful (Silva et al., 2009; Rudy, 2013; Bear et al., 2018a). In addition, current studies have merged this ECGi technology with computational models to provide patient-specific models in order to predict the efficacy of specific therapies (Boyle et al., 2018; Huntjens et al., 2018). Due to the influence of inhomogeneities inside the torso on the BSP, recent studies have also focused on the understanding of the forward problem and its relation with the inverse solution (Bear et al., 2015, 2018b; Zemzemi et al., 2015). Furthermore, the ill-posed nature of the problem requires different mathematical constraints and regularization methods to be used to find the most accurate physical and physiological solution (Oster and Rudy, 1992); recent studies have investigated the accuracy of these inverse methods (Bear et al., 2018b).

Despite these important works, there are still many questions in the field of ECGi which must be addressed in order to further develop the approach and improve its clinical and research impact. In this study, we provide analysis of the impact of electro-anatomical variability pertaining to differences in cardiac anatomy and heart rate on the accuracy of ECGi reconstructions obtained using different regularization methods. These analyses provide important insights for the interpretation of clinically obtained ECGi reconstructions over the time-course of an electro-anatomically dynamic condition such as heart failure.

\section{Wall Thickness and Heart Size}

Previous studies have investigated the influence of tissue inhomogeneities on the BSP and reconstructed solution, which were shown to have a small impact on the reconstructed signal (Ramanathan and Rudy, 2001; Zemzemi et al., 2015). 


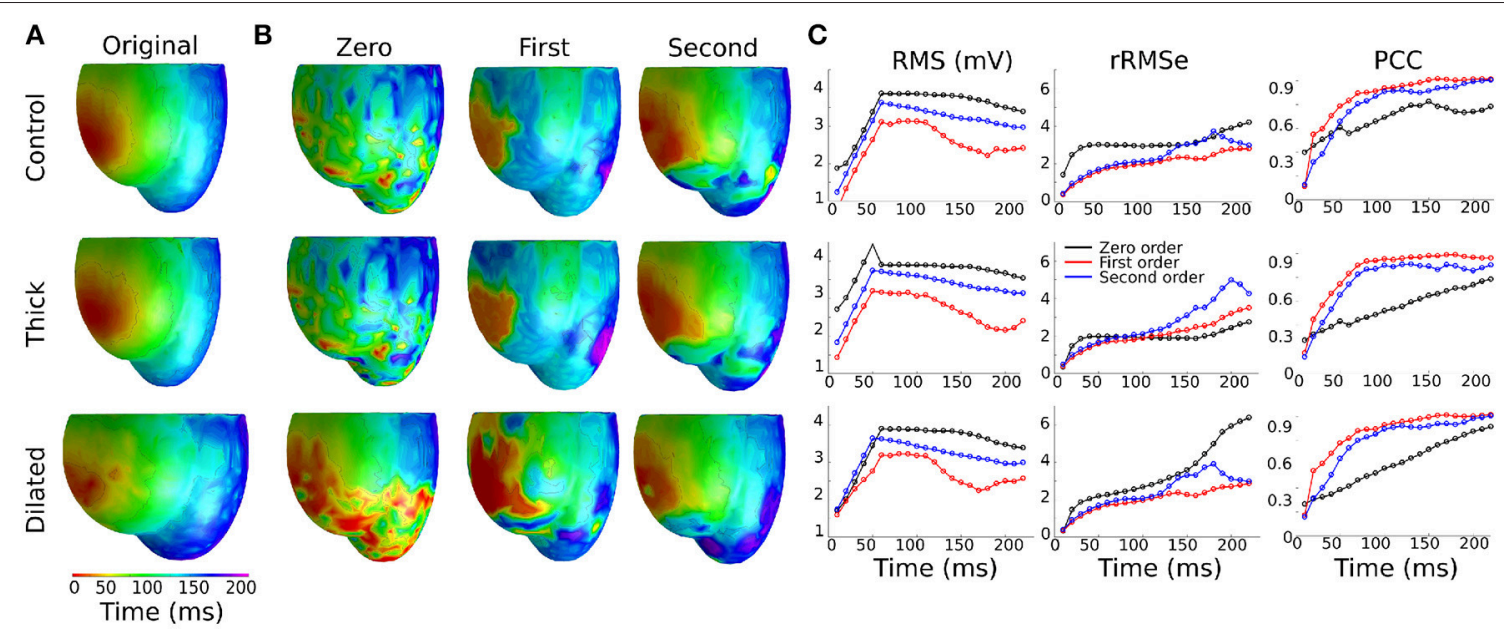

FIGURE 3 | Effects of heart geometry on the reconstructed epicardial potentials. (A) Original simulated ectopic activation initiated in the right ventricular lateral wall (RV-LAT) on all three geometries. (B) Reconstructed activation maps using Zero, First and Second order Tikhonov regularization using different geometries for the forward problem but the control/normal geometry for the inverse problem. Activation patterns were computed as the time of maximum negative first derivative $\mathrm{dV} / \mathrm{dt}$ at each location. (C) RMS, rRMSe, and PCC calculated to quantify the differences between the reconstructed activation patterns using Zero (black), First (blue) and Second (red) order Tikhonov regularization.

TABLE 2 | A summary of the effects of geometry on RMS, rRMSe, and PCC of reconstructed activation maps using the three different regularization methods.

\begin{tabular}{|c|c|c|c|c|}
\hline Tikhonov order & Metrics & $\begin{array}{l}\text { Control - mean } \\
\text { (SD) }\end{array}$ & $\begin{array}{l}\text { Dilated - mean } \\
\text { (SD) }\end{array}$ & $\begin{array}{c}\text { Thick - mean } \\
\text { (SD) }\end{array}$ \\
\hline \multirow[t]{2}{*}{ Zero order } & RMS & $3.45(0.60)$ & $3.74(0.43)$ & $3.65(0.62)$ \\
\hline & PCC & $0.673(0.108)$ & $0.570(0.152)$ & $0.603(0.120)$ \\
\hline First order & RMS & 3.03 (0.59) & $3.27(0.46)$ & $3.12(0.45)$ \\
\hline \multirow[t]{3}{*}{ Second order } & RMS & $2.48(0.59)$ & $2.68(0.48)$ & $2.63(0.44)$ \\
\hline & rRMSe & $1.97(0.64)$ & 2.65 (1.26) & $2.39(0.93)$ \\
\hline & PCC & $0.869(0.106)$ & $0.769(0.121)$ & $0.778(0.140)$ \\
\hline
\end{tabular}
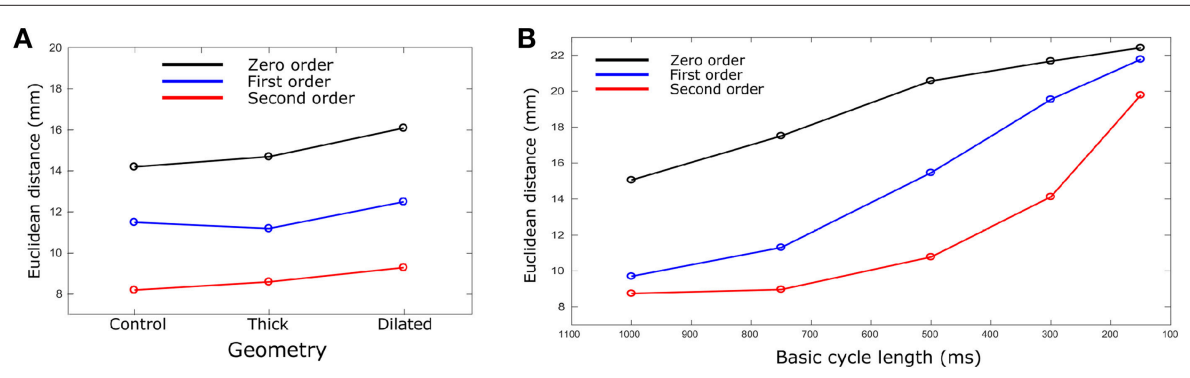

FIGURE 4 | Euclidean distance vs. (A) geometry and (B) BCL. Euclidean distances were calculated for each geometry case and BCL for Zero (black), First (Blue), and Second (red) order Tikhonov regularization. For (B), only control geometry was used. Data are the mean for all ectopic sites.

Effects such as an enlargement of the heart and thickening of the cardiac wall (associated with various disease states, e.g., heart failure) are not necessarily included in the geometrical transfer matrix, and have not been fully studied. In this study, we first compared the BSP activation maps obtained by modifying the size of the ventricles. This was used as the baseline comparison between BSP prior to obtaining the inverse solution. Using RMS, rRMSe, and PCC to quantify the similarity or differences between the BSP observed under these different conditions demonstrated that cardiac anatomy 

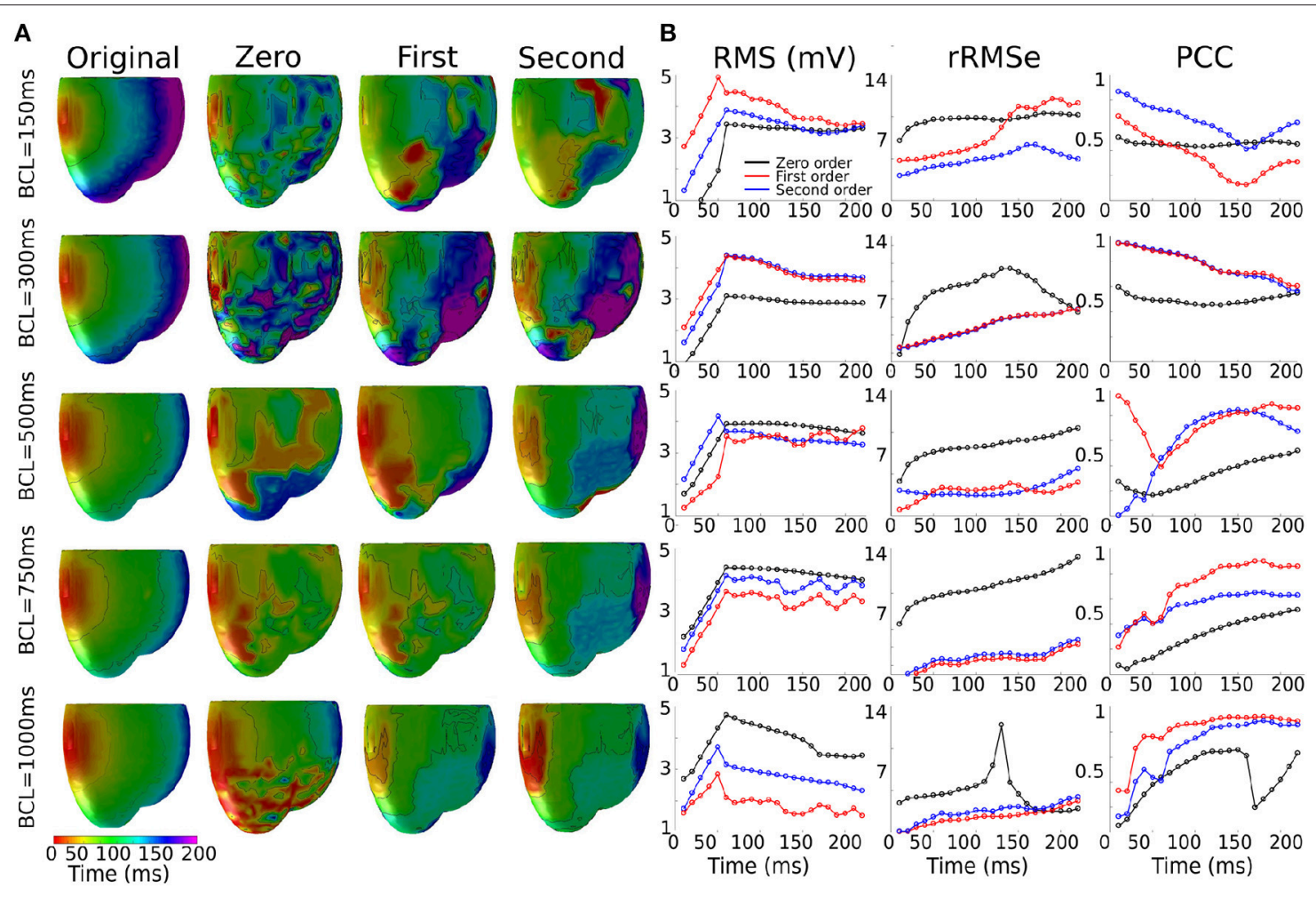

FIGURE 5 | Effects of the heart rate on the reconstructed activation maps. (A) 3D activation maps at different basic cycle lengths (BCLs): 150, 300, 500, 750, and 1,000 ms; activation time is given as the maximum negative slope of each local membrane potential. (B) RMS, rMSe and PCC measure calculated using Zero (black), First (blue), and Second (red) order Tikhonov regularization of an ectopic activation starting on the middle of the left ventricle (LV-LAT).

TABLE 3 | A summary of the effects of heart rate on reconstructed activation maps using the three different regularization methods.

\begin{tabular}{|c|c|c|c|c|c|c|}
\hline Tikhonov order & Metrics & BCL $1,000 \mathrm{~ms}$ & BCL 750 ms & BCL $500 \mathrm{~ms}$ & BCL $300 \mathrm{~ms}$ & BCL $150 \mathrm{~ms}$ \\
\hline \multirow[t]{3}{*}{ Zero order } & RMS & $3.85(0.55)$ & $3.96(0.62)$ & $3.53(0.65)$ & 3.67 (0.59) & 3.78 (1.02) \\
\hline & rRMSe & $7.64(2.3)$ & $7.81(1.75)$ & $8.31(1.41)$ & 8.77 (2.23) & $8.70(0.71)$ \\
\hline & PCC & $0.646(0.190)$ & $0.628(.154)$ & $0.626(0.119)$ & $0.629(0.103)$ & $0.623(0.107)$ \\
\hline \multirow[t]{3}{*}{ First order } & RMS & $2.74(0.39)$ & $3.61(0.61)$ & $4.16(0.66)$ & $4.59(0.63)$ & $4.20(0.61)$ \\
\hline & rRMSe & $2.82(0.96)$ & $2.94(1.01)$ & $3.14(0.93)$ & $4.91(1.46)$ & $4.92(1.06)$ \\
\hline & PCC & $0.820(0.169)$ & $0.789(0.106)$ & $0.757(0.280)$ & $0.713(0.122)$ & $0.686(0.130)$ \\
\hline \multirow[t]{3}{*}{ Second order } & RMS & $1.89(0.31)$ & $2.11(0.61)$ & $2.20(0.62)$ & $2.68(0.55)$ & $2.82(0.51)$ \\
\hline & rRMSe & $2.43(1.06)$ & $2.44(1.01)$ & $2.92(0.83)$ & $3.98(1.40)$ & $4.17(1.89)$ \\
\hline & PCC & $0.862(0.138)$ & 0.839 (0.213) & $0.813(0.148)$ & $0.789(0.107)$ & $0.705(0.160)$ \\
\hline
\end{tabular}

had a measurable effect on the details of the BSP but did not significantly alter the primary spatio-temporal features of normal activation (Figure 2).

Then, we observed how modifying the anatomy of the ventricles in the forward solution but not in the inverse approach had an effect on the accuracy of reconstructed ectopic activation. Larger RMS and rRMSe values were observed when comparing BSP error values vs. reconstructed error values (Figure 3; Table 2). Mostly, the first part of the activation (first $75 \mathrm{~ms}$ ) produced the most significant differences. However, it was still possible to identify the origin of ectopic activation, albeit with a small error (Figure 4A).
These results therefore indicate that it may not be necessary to repeat a cardiac CT/MRI when repeating ECGi in a patient who has undergone anatomical remodeling since their first ECGi procedure, which could significantly reduce the cost of long-term treatment. Some consideration may still be required to determine appropriate electrode positions-especially if the torso, as well as the heart, has undergone anatomical changes.

\section{Heart Rate}

Heart rate had a regularization approach-dependent effect on the accuracy of reconstructed activation patterns: In the case of the First and Second order the PCCs were larger at slow rates 
but significantly decreased when the heart rate was increased, exhibiting a negative temporal evolution over the activation period. In the case of Zero order, the values, even though not larger than the First or Second order in any case, remained similar when the heart rate increased and stable over the activation time at rapid rates (Figure 5; Table 3). The ED also showed an important dependency on the heart rate, increasing when the heart rate increased (Figure 4B). Therefore, these data indicate that the accuracy of inverse solutions in general decreases at rapid pacing rates. The underlying cause of this orderdependent difference in the rate-dependence of the solution is discussed in the next section, "On the Rate-Dependence of Time-Independent Solutions.”

Differences in the quality of reconstruction at fast rates may have particular clinical importance: higher rates may present the most clinically interesting results, for example exposing concealed abnormalities (Leong et al., 2018), yet produce the poorest reconstructions using ECGi. This may also indicate that rapid arrhythmias such as tachycardia or fibrillation could present the greatest challenges for reconstruction, additional to the spatial complexity of the excitation pattern itself.

\section{On the Rate-Dependence of Time-Independent Solutions}

We observed that the accuracy of the solution using both First and Second order methods was rate-dependent, resulting in larger errors and smaller correlations at rapid rates; a feature not observed using the Zero order method (Figures 4, 5; Table 3). This rate dependence raises an interesting question: given that the solutions to the inverse problem of electrocardiography use a quasi-static approximation, how does a temporal effect such as pacing rate modify the quality of the solution?

This can be explained by examining the differences between the regularization methods: A primary difference between the Zero order and First and Second order approaches is that the Zero order approach does not include any neighbor interaction (as it uses the identity matrix as the regularization operator) whereas the First and Second order do account for this interaction and result in smoothed signals (due to the use of the Gradient and Laplace operator for First and Second order, respectively). Spatial heterogeneities in voltage will therefore be smoothed using First and Second order but not Zero. Following that this approach distinction correlates with whether or not the reconstruction exhibits rate-dependence, we propose that spatial gradients observed at rapid rates, not present at slower pacing rates, may account for this observation.

In a previous preliminary study in the atria (Alday et al., 2016) we presented the hypothesis that this was primarily due to shortening of the AP morphology at rapid rates, resulting in a short excitation wavelength and therefore the simultaneous presence of both depolarization and repolarization wavefronts from a single excitation, significantly enhancing spatial gradients at temporal snapshots during the activation. An alternative explanation is that it is the presence of regions of tissue still active from the previous excitation at the time of the stimulus which lead to these enhanced spatial gradients. We tested which of these hypotheses was more likely to underlie the observation by comparing the control data at pacing cycle lengths of 300 and $1,000 \mathrm{~ms}$ with new simulations at those cycle lengths in which the AP duration has been significantly shortened (Figure 1Biii): this captures the shorter wavelength associated with rapid control pacing while simultaneously imposing that the previous excitation is no longer or only minimally present in the tissue at the time of excitation.

The RMS and PCC of First and Second order reconstructions associated with both the pacing rates using the short AP model were comparable to the slow pacing rate using the control AP model, and differed from the rapid pacing rates (Figure 6; Section S2 in Supplementary Material). In particular, the shorter AP models did not reproduce the negative temporal evolution and general lower PCC observed at the rapid rates using the control AP. These data indicate that, contrary to our original hypothesis, these increased errors were not caused by the short excitation wavelength (where it would be expected that all short AP models reproduced these features) but were rather caused by the presence of the previous excitation; the only condition which reproduced the lower correlation and large errors was the one in which large areas of still active tissue remained at the time of excitation (Figure 6C). The temporal evolution of the PCC also indicates that it is the presence of two large and distinct regions of active tissue, rather that multiple depolarization and repolarization wavefronts, which induces the reconstruction errors: the initial large PCC observed at rapid rates for control, which contrasts with the less accurate estimation of the focal location (i.e., increased $\mathrm{ED}$ ), is a result of accurate reconstruction of the large area of active tissue from the previous excitation; as the area of active tissue from the present excitation grows, the correlation decreases due to inaccurate reconstruction of two large regions. There is no large impact on the PCC at the time the previous excitation's depolarization wavefront terminates at full activation; rather, the lower correlation remains until the tissue repolarizes.

\section{Limitations}

These types of studies are key to fully translate ECGi technology into clinical settings. However, they are difficult or impossible to perform in control and experimental settings, and the accuracy of the forward and inverse solution is still under study (Bear et al., 2015, 2018b). Computational modeling offers an important tool to study, understand and provide insights into the effects of cardiac arrhythmias and clinical variables (Colman et al., 2013, 2017). Unfortunately, there are still several limitations that need to be addressed. The forward model lacks several inhomogeneities which may have an important effect on the BSP measured and therefore in the reconstructed signal, as we used a homogeneous torso approach for the inverse reconstruction. However, previous studies have shown that the effects of inhomogeneities in the inverse solution are small (Ramanathan and Rudy, 2001); (Zemzemi et al., 2015). 
A
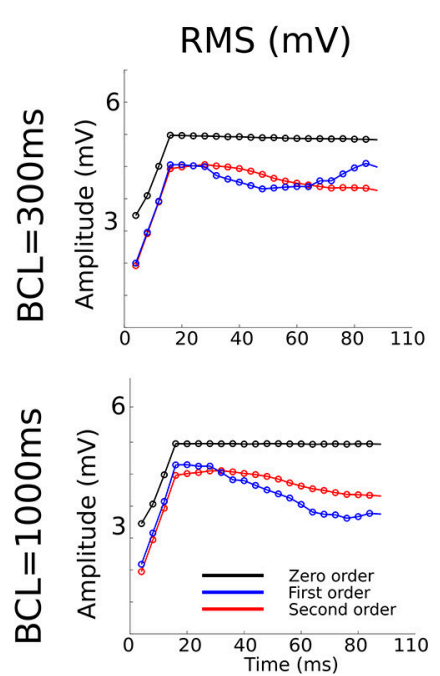

B

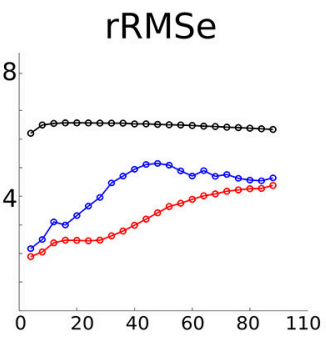

Short AP

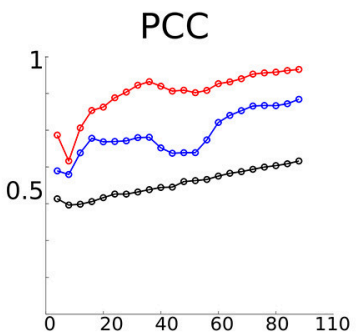

8
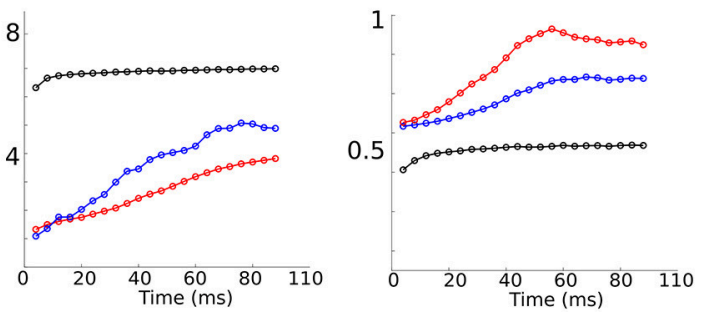

Normal AP
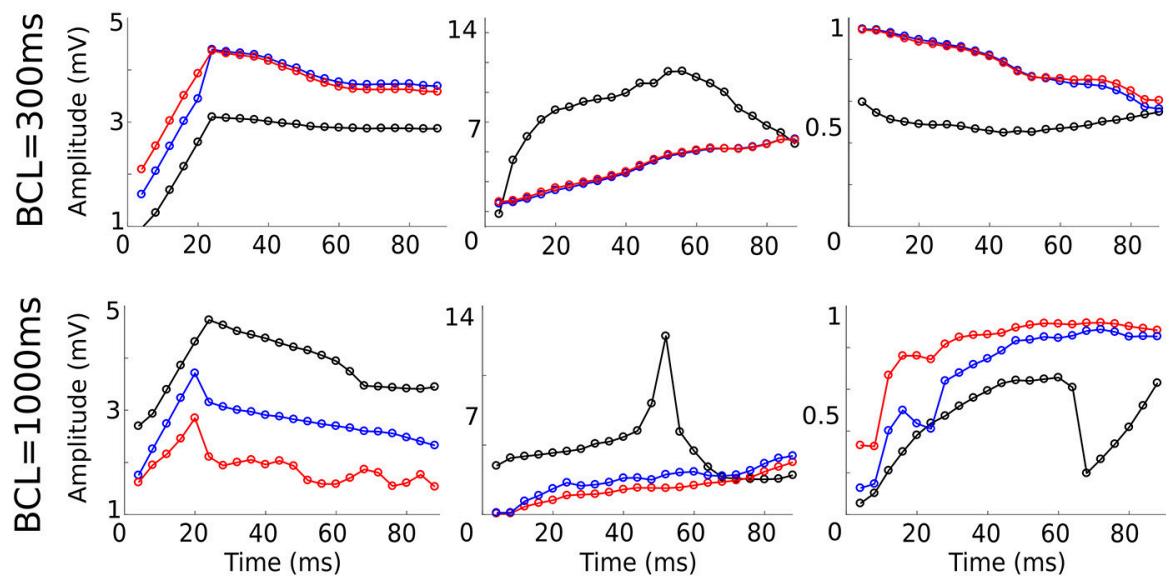

C

$$
t=30 \mathrm{~ms} \text { after stimulus }
$$

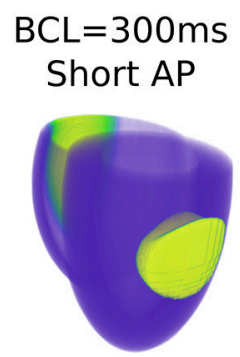

$\mathrm{BCL}=300 \mathrm{~ms}$
Normal AP

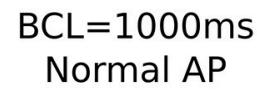

Short AP
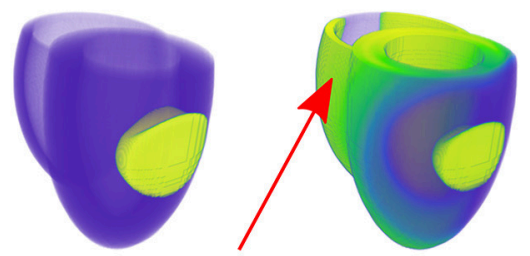

Previous

activation

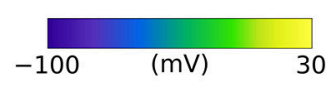

FIGURE 6 | RMS, rRMSe, and PCC of the reconstructed activation maps using (A) short and (B) normal AP durations at fast (BCL = 300 ms; upper panel) and slow $(\mathrm{BCL}=1,000 \mathrm{~ms}$; lower panel) pacing rates, for the Zero (black), First (blue), and Second (red) order Tikhonov regularization methods. (C) Corresponding snapshots of propagation at time $t=30 \mathrm{~ms}$ after stimulus of the LV-LAT ectopic site. The control geometry was used in all cases.

Another limitation is the idealized ventricular models used, which lacked the complex anatomy and microstructure of the real human ventricles (Stephenson et al., 2017). However, these were implemented to facilitate investigation of the effects of changing the size and wall thickness of the ventricles on non-invasive mapping. We used standard approaches to model cellular and 
ventricular electrophysiology, the general limitations of which have been addressed in detail elsewhere (Benson et al., 2011).

Due to both the BSP and inverse solution being computed using simulations, it is important to ensure that "inverse crime," where the inverse method exactly inverts the forward method, is avoided. We ensure that this is the case through the use of Salu's method for the forward problem and Barr's for the inverse solution; thus, whereas the forward problem is solved by computing the electric field which arises as a result of currents in the cardiac tissue, the inverse solution uses an equivalent potential distribution on a closed surface. These independent methods, utilizing two different matrices, ensure that it is not possible for the inverse solution to exactly invert the forward solution.

Only ventricular activation was considered for comparison between different rates and geometries, disregarding potential analysis of the repolarization patterns, which may themselves provide substantial diagnostic information. Future investigation of the effects of anatomical reconstruction inaccuracies and heart rate on the reconstruction of repolarization patterns may therefore provide valuable information. However, the present study was focused on identifying the location of ectopic pacing sites, relevant in particular for guiding ablation therapy, and therefore requires only activation patterns to be reconstructed.

There are multiple further factors which will be relevant for clinical studies but not accounted for in the idealized and controlled in silico experiments of the present study. Whereas the geometry of the heart was considered, this was not combined with analysis of its location, its mechanical movement, or electrode location errors, all of which have been previously shown to be important factors influencing the accuracy of the reconstruction (Swenson et al., 2011; Cluitmans and Volders, 2017; Cluitmans et al., 2017; Coll-Font and Brooks, 2018). Furthermore, we did not investigate whether the effect of heart rate was influenced by the geometry, treating these analyses as separate; such investigation may provide further important insight.

In addition, the inverse problem of electrocardiography is an ill-posed problem and therefore noisy signals can have an important effect of the reconstructed maps. Whereas, the simulated data in this study and used for our analyses did not include noise, we performed further simulations in which white noise was included. These data demonstrated that noise increased ED and decreased correlation, but the differences between conditions were maintained, indicating that whereas noise has an important impact on the activation maps and ED, our observations about different geometries and pacing rate are maintained (Section S3 in Supplementary Material; Figures S4-S7, and Tables S1, S2). Also, the results obtained in this study are of the same order of magnitude observed in previous studies (Wang et al., 2010; Bear et al., 2018b); (Tate et al., 2018).

\section{REFERENCES}

Adeniran, I., McPate, M. J., Witchel, H. J., Hancox, J. C., and Zhang, H. (2011). Increased vulnerability of human ventricle to re-entrant excitation in

\section{CONCLUSION}

The systematic analysis revealed that the effect of size, thickness, and heart rate can manifest in the BSP and ECGi in different ways, with varying sensitivities and success rates in inferring the clinical variables from non-invasive information. We observed a rate dependence in the ability of different Tikhonov regularization methods to successfully reproduce cardiac electrical activity. Our results show that the ECGi approach gives the most accurate results when used with geometries depicting the current state of the patient's heart, but if a single image of the patient's heart is obtained, for example at the start of treatment, the ECGi approach still gives useful and reasonably accurate information relating to underlying electrophysiological abnormalities. In addition, clinical variables such as heart rate need to be accounted for when solving the inverse solution, in particular due to the increase in errors observed at rapid pacing rates.

\section{AUTHOR CONTRIBUTIONS}

EP conceived the study. EP and MC designed the study. DW developed, applied, and analyzed data associated with the bi-ventricle cardiac excitation model. EP developed, applied, and analyzed data associated with the forward problem, inverse solution, ECG, and error analysis. EP and all other authors interpreted the data. EP and DW prepared the figures. EP and MC prepared, and all authors edited, the drafts of the manuscript. All authors approve the final version of the manuscript and agreed to be accountable for all aspects of the work in ensuring that questions related to the accuracy and integrity of the work are appropriately investigated and resolved.

\section{FUNDING}

This work is supported by a Wellcome Trust ISSF Fellowship to DW (204825/Z/16/Z) (Wellcome Trust ISSF Fellow-University of Leeds account), a British Heart Foundation project grant to AB (PG/16/74/32374) and a Medical Research Council Strategic Skills Fellowship to MC (MR/M014967/1).

\section{ACKNOWLEDGMENTS}

Parts of this work were undertaken on ARC3, part of the High Performance Computing facilities at the University of Leeds, UK.

\section{SUPPLEMENTARY MATERIAL}

The Supplementary Material for this article can be found online at: https://www.frontiersin.org/articles/10.3389/fphys. 2019.00308/full\#supplementary-material

hERG-linked variant 1 short QT syndrome. PLoS Comput. Biol. 7:e1002313. doi: 10.1371/journal.pcbi.1002313

Adeniran, I., Whittaker, D. G., El Harchi, A., Hancox, J. C., and Zhang, H. (2017). In silico investigation of a KCNQ1 mutation associated 
with short QT syndrome. Sci. Rep. 7:8469. doi: 10.1038/s41598-01708367-2

Alday, E. A., Ni, H., Zhang, C., Colman, M. A., Gan, Z., and Zhang, H. (2016). Comparison of electric- and magnetic-cardiograms produced by myocardial ischemia in models of the human ventricle and torso. PLoS ONE 11:e0160999. doi: 10.1371/journal.pone.0160999

Alfakih, K., Plein, S., Thiele, H., Jones, T., Ridgway, J. P., and Sivananthan, M. U. (2003). Normal human left and right ventricular dimensions for MRI as assessed by turbo gradient echo and steady-state free precession imaging sequences. J. Magn. Reson. Imaging 17, 323-329. doi: 10.1002/jmri.10262

Appel, M. L., Berger, R. D., Saul, J. P., Smith, J. M., and Cohen, R. J. (1989). Beat to beat variability in cardiovascular variables: noise or music? J. Am. Coll. Cardiol. 14, 1139-1148. doi: 10.1016/0735-1097(89)90408-7

Barr, R. C., Ramsey, M., and Spach, M. S. (1977). Relating epicardial to body surface potential distributions by means of transfer coefficients based on geometry measurements. IEEE Trans. Biomed. Eng. 24, 1-11. doi: 10.1109/TBME.1977.326201

Baumert, M., Javorka, M., and Kabir, M. M. (2013). Joint symbolic analyses of heart rate, blood pressure, and respiratory dynamics. J. Electrocardiol. 46, 569-573. doi: 10.1016/j.jelectrocard.2013.07.009

Bear, L., Huntjens, P., Coronel, R., Bernus, O., Dallet, C., Walton, R., et al. (2016). "Detection of incomplete left bundle branch block by non-invasive electrocardiographic imaging," in Computing in Cardiology Conference (CinC), 2016 (Vancouver, BC: IEEE), 389-392.

Bear, L. R., Cheng, L. K., Legrice, I. J., Sands, G. B., Lever, N. A., Paterson, D. J., et al. (2015). Forward problem of electrocardiography: is it solved? Circ. Arrhythm. Electrophysiol. 8, 677-684. doi: 10.1161/CIRCEP.114.001573

Bear, L. R., Huntjens, P. R., Walton, R. D., Bernus, O., Coronel, R., and Dubois, R. (2018a). Cardiac electrical dyssynchrony is accurately detected by noninvasive electrocardiographic imaging. Heart Rhythm 15, 1058-1069. doi: 10.1016/j.hrthm.2018.02.024

Bear, L. R., Legrice, I. J., Sands, G. B., Lever, N. A., Loiselle, D. S., Paterson, D. J., et al. (2018b). How accurate is inverse electrocardiographic mapping? A systematic in vivo evaluation. Circ. Arrhythm. Electrophysiol. 11:e006108. doi: 10.1161/CIRCEP.117.006108

Benjamin, E. J., Blaha, M. J., Chiuve, S. E., Cushman, M., Das, S. R., Deo, R., et al. (2017). Heart Disease and Stroke Statistics-2017 update: a report from the American Heart Association. Circulation 135, e146-e603. doi: 10.1161/CIR.0000000000000485

Benson, A., Gilbert, S., Li, P., Newton, S., and Holden, A. (2008). Reconstruction and quantification of diffusion tensor imaging-derived cardiac fibre and sheet structure in ventricular regions used in studies of excitation propagation. Math. Modell. Nat. Phenomena 3, 101-130. doi: 10.1051/mmnp:2008083

Benson, A. P., Al-Owais, M., and Holden, A. V. (2011). Quantitative prediction of the arrhythmogenic effects of de novo hERG mutations in computational models of human ventricular tissues. Eur. Biophys. J. 40, 627-639. doi: 10.1007/s00249-010-0663-2

Benson, A. P., Bernus, O., Dierckx, H., Gilbert, S. H., Greenwood, J. P., Holden, A. V., et al. (2010). Construction and validation of anisotropic and orthotropic ventricular geometries for quantitative predictive cardiac electrophysiology. Interface Focus. 1, 101-116. doi: 10.1098/rsfs.2010.0005

Benson, A. P., Halley, G., Li, P., Tong, W. C., and Holden, A. V. (2007). Virtual cell and tissue dynamics of ectopic activation of the ventricles. Chaos 17:015105. doi: 10.1063/1.2404634

Boukens, B. J., Sulkin, M. S., Gloschat, C. R., Ng, F. S., Vigmond, E. J., and Efimov, I. R. (2015). Transmural APD gradient synchronizes repolarization in the human left ventricular wall. Cardiovasc. Res. 108, 188-196. doi: 10.1093/cvr/cvv202

Boyle, P. M., Hakim, J. B., Zahid, S., Franceschi, W. H., Murphy, M. J., Vigmond, E. J., et al. (2018). Comparing reentrant drivers predicted by image-based computational modeling and mapped by electrocardiographic imaging in persistent atrial fibrillation. Front. Physiol. 9:414. doi: 10.3389/fphys.2018.00414

Clay, S., Alfakih, K., Radjenovic, A., Jones, T., and Ridgway, J. P. (2006). Normal range of human left ventricular volumes and mass using steady state free precession MRI in the radial long axis orientation. Magn. Reson. Mater. Phys. Biol. Med. 19, 41-45. doi: 10.1007/s10334-005-0025-8

Cluitmans, M. J., Bonizzi, P., Karel, J. M., Das, M., Kietselaer, B. L., De Jong, M. M., et al. (2017). In vivo validation of electrocardiographic imaging. JACC Clin. Electrophysiol. 3, 232-242. doi: 10.1016/j.jacep.2016.11.012
Cluitmans, M. J., and Volders, P. G. (2017). Influence of body-surface geometry accuracy on noninvasive reconstruction of electrical activation and recovery in electrocardiographic imaging. Computing 44:1. doi: 10.22489/CinC.2017.243-189

Coll-Font, J., and Brooks, D. (2018). Tracking the position of the heart from body surface potential maps and electrograms. Front. Physiol. 9:1727. doi: 10.3389/fphys.2018.01727

Colman, M. A., Aslanidi, O. V., Kharche, S., Boyett, M. R., Garratt, C., Hancox, J. C., et al. (2013). Pro-arrhythmogenic effects of atrial fibrillation-induced electrical remodelling: insights from the three-dimensional virtual human atria. J. Physiol. 591, 4249-4272. doi: 10.1113/jphysiol.2013.254987

Colman, M. A., Perez Alday, E. A., Holden, A. V., and Benson, A. P. (2017). Trigger vs. substrate: multi-dimensional modulation of QT-prolongation associated arrhythmic dynamics by a hERG channel activator. Front. Physiol. 8:757. doi: 10.3389/fphys.2017.00757

Dubois, R., Shah, A. J., Hocini, M., Denis, A., Derval, N., Cochet, H., et al. (2015). Non-invasive cardiac mapping in clinical practice: application to the ablation of cardiac arrhythmias. J. Electrocardiol. 48, 966-974. doi: 10.1016/j.jelectrocard.2015.08.028

Durrer, D., Van Dam, R. T., Freud, G., Janse, M., Meijler, F., and Arzbaecher, R. (1970). Total excitation of the isolated human heart. Circulation 41, 899-912. doi: 10.1161/01.CIR.41.6.899

Gage, R. M., Curtin, A. E., Burns, K. V., Ghosh, S., Gillberg, J. M., and Bank, A. J. (2017). Changes in electrical dyssynchrony by body surface mapping predict left ventricular remodeling in patients with cardiac resynchronization therapy. Heart Rhythm 14, 392-399. doi: 10.1016/j.hrthm.2016.11.019

Ghosh, S., Silva, J. N., Canham, R. M., Bowman, T. M., Zhang, J., Rhee, E. K., et al. (2011). Electrophysiologic substrate and intraventricular left ventricular dyssynchrony in nonischemic heart failure patients undergoing cardiac resynchronization therapy. Heart Rhythm 8, 692-699. doi: 10.1016/j.hrthm.2011.01.017

Glukhov, A. V., Fedorov, V. V., Lou, Q., Ravikumar, V. K., Kalish, P. W. Schuessler, R. B., et al. (2010). Transmural dispersion of repolarization in failing and nonfailing human ventricle. Circ. Res. 106, 981-991. doi: 10.1161/CIRCRESAHA.109.204891

Goldenberg, I., Moss, A. J., and Zareba, W. (2006). QT interval: how to measure it and what is "normal". J. Cardiovasc. Electrophysiol. 17, 333-336. doi: $10.1111 /$ j.1540-8167.2006.00408.x

Hansen, P. C. (1992). Analysis of discrete ill-posed problems by means of the L-curve. SIAM Rev. 34, 561-580. doi: 10.1137/1034115

Hansen, P. C. (1998). Rank-Deficient and Discrete Ill-Posed Problems: Numerical Aspects of Linear Inversion. SIAM.

Ho, S., and Nihoyannopoulos, P. (2006). Anatomy, echocardiography, and normal right ventricular dimensions. Heart 92, i2-i13. doi: 10.1136/hrt.2005.077875

Ho, S. Y. (2009). Anatomy and myoarchitecture of the left ventricular wall in normal and in disease. Eur. J. Echocardiogr. 10, iii3-iii7. doi: 10.1093/ejechocard/jep159

Huntjens, P. R., Ploux, S., Strik, M., Walmsley, J., Ritter, P., Haissaguerre, M., et al. (2018). Electrical substrates driving response to cardiac resynchronization therapy: a combined clinical-computational evaluation. Circ. Arrhythm. Electrophysiol. 11:e005647. doi: 10.1161/CIRCEP.117.005647

Kabir, M. M., Sedaghat, G., Thomas, J., Waks, J. W., Sitlani, C. M., Biggs, M. L., et al. (2016). Electrocardiographic beat-to-beat QT interval is affected by phases of respiration: the atherosclerosis risk in communities study. Am. Heart Assoc. 134, A12224-A12224. Available online at: https://www.ahajournals.org/doi/ abs/10.1161/circ.134.suppl_1.12224

Keller, D. U., Jarrousse, O., Fritz, T., Ley, S., Dossel, O., and Seemann, G. (2011). Impact of physiological ventricular deformation on the morphology of the T-wave: a hybrid, static-dynamic approach. IEEE Trans. Biomed. Eng. 58, 2109-2119. doi: 10.1109/TBME.2011.2147785

Langley, P., Bowers, E. J., and Murray, A. (2010). Principal component analysis as a tool for analyzing beat-to-beat changes in ECG features: application to ECG-derived respiration. IEEE Trans. Biomed. Eng. 57, 821-829. doi: 10.1109/TBME.2009.2018297

Leong, K. M., Ng, F. S., Roney, C., Cantwell, C., Shun-Shin, M. J., Linton, N. W., et al. (2018). Repolarization abnormalities unmasked with exercise in sudden cardiac death survivors with structurally normal hearts. J. Cardiovasc. Electrophysiol. 29, 115-126. doi: 10.1111/jce.13375 
Macfarlane, P. W., van Oosterom, A., Pahlm, O., Kligfield, P., Janse, M., and Camm, J. (eds) (2010). Comprehensive Electrocardiology. London: Springer Science \& Business Media.

Oster, H. S., and Rudy, Y. (1992). The use of temporal information in the regularization of the inverse problem of electrocardiography. IEEE Trans. Biomed. Eng. 39, 65-75. doi: 10.1109/10.108129

Perez Alday, E. A., Michael, A. C., and Henggui, Z. (2016). "Reconstruction of atrial ectopic focal and re-entrant excitations from body surface potentials. Insights from 3D virtual human atria and torso," in 2016 Computing in Cardiology Conference (CinC), (Vancouver, BC: IEEE). doi: 10.23919/CIC.2016. 7868839

Perez-Alday, E. A., Colman, M. A., Langley, P., Butters, T. D., Higham, J., Workman, A. J., et al. (2015). A new algorithm to diagnose atrial ectopic origin from multi lead ECG systems-insights from 3D virtual human atria and torso. PLoS Comput. Biol. 11:e1004026. doi: 10.1371/journal.pcbi. 1004026

Perez-Alday, E. A., Colman, M. A., Langley, P., and Zhang, H. (2017a). Novel noninvasive algorithm to identify the origins of re-entry and ectopic foci in the atria from 64-lead ECGs: a computational study. PLoS Comput. Biol. 13:e1005270. doi: 10.1371/journal.pcbi.1005270

Perez-Alday, E. A., Thomas, J. A., Kabir, M., Sedaghat, G., Rogovoy, N., Van Dam, E., et al. (2017b). Torso geometry reconstruction and body surface electrode localization using three-dimensional photography. J. Electrocardiol. 51, 60-67. doi: 10.1016/j.jelectrocard.2017.08.050

Ramanathan, C., and Rudy, Y. (2001). Electrocardiographic imaging: II. Effect of torso inhomogeneities on noninvasive reconstruction of epicardial potentials, electrograms, and isochrones. J. Cardiovasc. Electrophysiol. 12, 241-252. doi: 10.1046/j.1540-8167.2001.00241.x

Rodrigo, M., Climent, A. M., Liberos, A., Fernández-Avilés, F., Berenfeld, O., Atienza, F., et al. (2017). Technical considerations on phase mapping for identification of atrial reentrant activity in direct-and inversecomputed electrograms. Circ. Arrhythm. Electrophysiol. 10:e005008. doi: 10.1161/CIRCEP.117.005008

Rudy, Y. (2013). Noninvasive electrocardiographic imaging of arrhythmogenic substrates in humans. Circ. Res. 112, 863-874. doi: 10.1161/CIRCRESAHA.112.279315

Salu, Y. (1980). Implementing a consistency criterion in numerical solution of the bioelectric forward problem. IEEE Trans. Biomed. Eng. 338-341. doi: 10.1109/TBME.1980.326742

Seemann, G., Keller, D., Weiss, D., and Dossel, O. (2006). "Modeling human ventricular geometry and fiber orientation based on diffusion tensor MRI", in Computers in Cardiology, 2006 (IEEE), 801-804. Available online at: https:// ieeexplore.ieee.org/abstract/document/4511973

Shimizu, I., and Minamino, T. (2016). Physiological and pathological cardiac hypertrophy. J. Mol. Cell. Cardiol. 97, 245-262. doi: 10.1016/j.yjmcc.2016.06.001

Silva, J. N., Ghosh, S., Bowman, T. M., Rhee, E. K., Woodard, P. K., and Rudy, Y. (2009). Cardiac resynchronization therapy in pediatric congenital heart disease: insights from noninvasive electrocardiographic imaging. Heart Rhythm 6, 1178-1185. doi: 10.1016/j.hrthm.2009. 04.017

Stephenson, R. S., Atkinson, A., Kottas, P., Perde, F., Jafarzadeh, F., Bateman, M., et al. (2017). High resolution 3-dimensional imaging of the human cardiac conduction system from microanatomy to mathematical modeling. Sci. Rep. 7:7188. doi: 10.1038/s41598-017-07694-8

Streeter, D. D., Spotnitz, H. M., Patel, D. P., Ross, J., and Sonnenblick, E. H. (1969). Fiber orientation in the canine left ventricle during diastole and systole. Circ. Res. 24, 339-347. doi: 10.1161/01.RES.24.3.339
Swenson, D. J., Geneser, S. E., Stinstra, J. G., Kirby, R. M., and Macleod, R. S. (2011) Cardiac position sensitivity study in the electrocardiographic forward problem using stochastic collocation and boundary element methods. Ann. Biomed. Eng. 39:2900. doi: 10.1007/s10439-011-0391-5

Szabó, G., Szentandrássy, N., Bíró, T., Tóth, B. I., Czifra, G., Magyar, J., et al. (2005). Asymmetrical distribution of ion channels in canine and human left-ventricular wall: epicardium versus midmyocardium. Pflügers Arch. 450, 307-316. doi: 10.1007/s00424-005-1445-z

Szentadrassy, N., Banyasz, T., Biro, T., Szabo, G., Toth, B. I., Magyar, J., et al. (2005). Apico-basal inhomogeneity in distribution of ion channels in canine and human ventricular myocardium. Cardiovasc. Res. 65, 851-860. doi: 10.1016/j.cardiores.2004.11.022

Taggart, P., Sutton, P. M., Opthof, T., Coronel, R., Trimlett, R., Pugsley, W., et al. (2000). Inhomogeneous transmural conduction during early ischaemia in patients with coronary artery disease. J. Mol. Cell. Cardiol. 32, 621-630. doi: 10.1006/jmcc.2000.1105

Tate, J., Gillette, K., Burton, B., Good, W., Zenger, B., Coll-Font, J., et al. (2018). Reducing error in ECG forward simulations with improved source sampling. Front. Physiol. 9:1304. doi: 10.3389/fphys.2018.01304

Ten Tusscher, K. H., and Panfilov, A. V. (2006). Alternans and spiral breakup in a human ventricular tissue model. Am. J. Physiol. Heart Circ. Physiol. 291, H1088-H1100. doi: 10.1152/ajpheart.00109.2006

Vriesendorp, P. A., Schinkel, A. F., Liebregts, M., Theuns, D. A., Van Cleemput, J., Folkert, J., et al. (2015). Validation of the 2014 European Society of Cardiology guidelines risk prediction model for the primary prevention of sudden cardiac death in hypertrophic cardiomyopathy. Circ. Arrhythm. Electrophysiol. 8, 829-835. doi: 10.1161/CIRCEP.114.002553

Wang, D., Kirby, R., Macleod, R. S., and Johnson, C. A. (2010). "New family of variational-form-based regularizers for reconstructing epicardial potentials from body-surface mapping," in Computing in Cardiology, 2010 (Belfast, UK: IEEE), 93-96.

Whittaker, D. G., Ni, H., Benson, A. P., Hancox, J. C., and Zhang, H. (2017). Computational analysis of the mode of action of disopyramide and quinidine on hERG-linked short QT syndrome in human ventricles. Front. Physiol. 8:759. doi: 10.3389/fphys.2017.00759

Zemzemi, N., Dobrzynski, C., Bear, L., Potse, M., Dallet, C., Coudière, Y., et al. (2015). "Effect of the torso conductivity heterogeneities on the ECGI inverse problem solution," in Computing in Cardiology Conference (CinC), 2015 (Nice: IEEE), 233-236. doi: 10.1109/CIC.2015.7408629

Zhang, J., Cooper, D. H., Desouza, K. A., Cuculich, P. S., Woodard, P. K., Smith, T. W., et al. (2016). Electrophysiologic scar substrate in relation to VT: noninvasive high-resolution mapping and risk assessment with ECGI. Pacing Clin. Electrophysiol. 39, 781-791. doi: 10.1111/pace.12882

Conflict of Interest Statement: The authors declare that the research was conducted in the absence of any commercial or financial relationships that could be construed as a potential conflict of interest.

The reviewer JT declared a past collaboration with one of the authors EP to the handling editor.

Copyright (c) 2019 Perez Alday, Whittaker, Benson and Colman. This is an openaccess article distributed under the terms of the Creative Commons Attribution License (CC BY). The use, distribution or reproduction in other forums is permitted, provided the original author(s) and the copyright owner(s) are credited and that the original publication in this journal is cited, in accordance with accepted academic practice. No use, distribution or reproduction is permitted which does not comply with these terms. 